\title{
Grain boundary contribution to recoil loop openness of exchange-coupled nanocrystalline magnets
}

\author{
Chuan-bing Ronga) and J. Ping Liu \\ Department of Physics, University of Texas at Arlington, Arlington, Texas 76019, USA
}

(Received 3 March 2009; accepted 12 April 2009; published online 29 April 2009)

\begin{abstract}
It has been well known that recoil loop openness is related to soft-phase presence in exchange-coupled hard-soft nanocomposite magnets. Our study on recoil loop openness of exchange-coupled nanocrystalline magnets (both single-phase and composite) using a micromagnetic finite-element method has revealed that the recoil loop openness is also due to decreased grain size. Open recoil loops exist in single-phase magnets as well. Simulation of magnetization distribution in both nanocrystalline single-phase magnets and nanocomposite magnets shows that the openness of the recoil loops is correlated with unstable magnetization behavior in grain boundary and soft-phase regions, which is attributed to high energy state caused by exchange coupling in these regions. The simulation results are supported by experimental data. (C) 2009 American Institute of Physics. [DOI: 10.1063/1.3127226]
\end{abstract}

Magnetically hard/soft exchange-coupled nanocomposites show enhanced magnetic properties due to intergrain exchange interactions. ${ }^{1-3}$ Strength of the intergranular exchange coupling in these types of materials can be evaluated by measuring the remanence ratio or recoil loops. Analysis of recoil loops can provide deep physical insight into magnetization reversal behavior in nanocomposite magnets. ${ }^{4-13}$ However, physical origin of the open recoil loops in nanocrystalline magnets is still not well understood. ${ }^{11-13}$ It is commonly accepted that the open recoil loop phenomena were resulted from exchange decoupled soft phase in a nanocomposite magnet. ${ }^{4-10}$ Recently, the recoil loop openness was found to be related to the inhomogeneity in the magnetic anisotropy, ${ }^{11}$ unstable magnetic moments affected by thermal fluctuation, ${ }^{12}$ and variations of exchange interactions in nanocomposite magnets. ${ }^{13}$ It seems that the origin of open recoil loops in nanocrystalline magnets is more complex than what was previously thought and needs to be further studied.

Micromagnetic simulation is an effective method to study magnetization behaviors inside nanostructured magnets since it gives a clear physics explanation on mechanisms of magnetization distribution and reversal. ${ }^{14-19}$ In this work, we extend our study from nanocomposite magnets to singlephase magnets as well, to investigate the origin of recoil loop openness in exchange-coupled nanocrystalline magnets by using a micromagnetic finite-element method. It is revealed that not only the soft phases but also grain boundaries contribute to openness of the recoil loops.

The equilibrium magnetization distribution under external magnetic field is calculated by the minimization of the total magnetic Gibbs free energy. It should be mentioned here that a magnetic vector potential was introduced to calculate the upper bound of the stray field energy, which was suggested by Brown. ${ }^{20}$ The resulting open-boundary problem can be treated by a parallelepipedic shell transformation, which transforms the infinite exterior space of the magnet on a shell around the magnet. ${ }^{21} \mathrm{~A}$ brief description of the micromagnetic finite-element method used to discrete the magnets

${ }^{a)}$ Electronic mail: crong@uta.edu. was reported in our previous works ${ }^{3,17-19}$ and more details can be found in the works by Schrefl $e t a .^{22}$ and Fisher et $a l^{15,23} \mathrm{An}$ isotropic single-phase magnet was constructed by assembling of 64 cubic $\mathrm{Nd}_{2} \mathrm{Fe}_{14} \mathrm{~B}$ grains with random orientation, as shown in the inset of Fig. 1(a). A hard-soft phase nanocomposite magnet was constructed by filling the magnetically soft $\alpha$-Fe phase into the space between the $\mathrm{Nd}_{2} \mathrm{Fe}_{14} \mathrm{~B}$ hard-phase grains, as shown in the inset of Fig. 1(b). The size (edge length $L$ ) of the $\mathrm{Nd}_{2} \mathrm{Fe}_{14} \mathrm{~B}$ grains varies from 10 to $50 \mathrm{~nm}$, while the thickness of the $\alpha$-Fe layers $(t)$ varies from 0 to $6 \mathrm{~nm}$. The material parameters of the two phases used in this work were adopted from Ref. 14.

Figure 1 shows a simulated recoil loop of the singlephase sample $(L=20 \mathrm{~nm})$ and the nanocomposite sample $(L=20 \mathrm{~nm}$ and $t=3 \mathrm{~nm}$ ). The recoil loop openness will be defined and quantitatively discussed later. We will also see that recoil loops with their tips at coercive fields have the maximum openness. It is striking to see that the recoil loops of a single-phase magnet are also open, though the openness of the simulated nanocomposite magnet is larger than that of a single-phase sample. This result indicates that the open recoil loops are not only related to a soft phase in a nanocomposite but also to grain size of the nanocrystalline magnet. For years, the phenomenon of open recoil loops is considered only as a result of soft-phase effect in a composite magnet, which is probably related to the fact that grain size in conventional single-phase magnets is usually very large compared to the size range studied for recoil loop phenomenon in this work.

To quantitatively describe a recoil loop, we use $\Delta J_{\mathrm{rc}}(H)=\left[J_{\mathrm{up}}(H)-J_{\mathrm{dw}}(H)\right]$ as its openness where $J_{\text {up }}(H)$ and $J_{\mathrm{dw}}(H)$ are the magnetization values of the upper and lower branches on the recoil loops under an applied field $H$. The maximum $\Delta J_{\mathrm{rc}}(H)$ value in each recoil loop (with maximum reversing field $\left.H_{R}\right)$ was defined as $\Delta J_{\mathrm{rc}}^{m}\left(H_{R}\right)$. The detailed definition can be found in Refs. 12 and 24. Figure 2(a) shows the dependence of $\Delta J_{\mathrm{rc}}^{m}\left(H_{R}\right)$ on $H_{R}$ of the single-phase magnets with different grain size. The high peak value in the $\Delta \mathrm{J}_{\mathrm{rc}}^{m}\left(H_{R}\right) \sim H_{R}$ curves means a large recoil loop openness. Interestingly, it has been observed that the recoil loop open- 

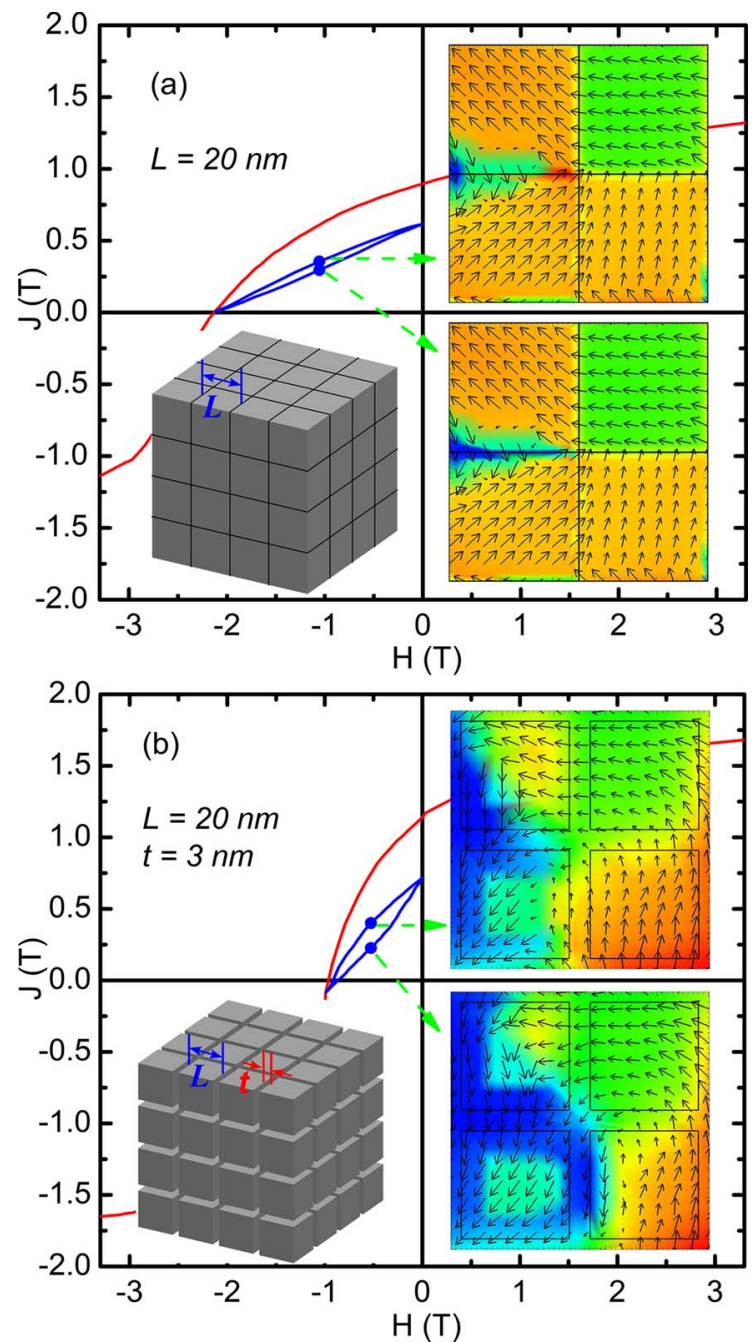

FIG. 1. (Color online) The recoil loops with maximum openness of (a) the single-phase magnet with $L=20 \mathrm{~nm}$ and (b) the nanocomposite magnet with $L=20 \mathrm{~nm}$ and $t=3 \mathrm{~nm}$. The insets shown in the third quadrant are the three-dimensional model of single-phase and hard-soft phase nanocomposite magnets. $L$ and $t$ represent the grain size of $\mathrm{Nd}_{2} \mathrm{Fe}_{14} \mathrm{~B}$ phase and the thickness of soft $\alpha$-Fe phase layer. The insets in the right side give the magnetization distributions at different magnetic history, i.e., upper and lower branches of recoil loop under field $H=H_{R} / 2$. The color red and blue (light and dark in print version) in the distributions represent the magnetic moments with deviation to up and down, respectively. To illustrate the magnetization distributions clearly, the slice plane of only four grains was selected instead of the whole sample.

ness increases with decreasing grain size, indicating that the intergranular exchange-coupling interactions in single-phase magnets also play an important role in the occurrence of open recoil loops. To verify the simulated result, Fig. 3 shows the recoil loops of ball-milled $\mathrm{Nd}_{2} \mathrm{Fe}_{14} \mathrm{~B}$ single-phase magnets with different grain size. The $\mathrm{Nd}_{2} \mathrm{Fe}_{14} \mathrm{~B}$ nanocrystalline powders (the particle size is about $20-50 \mu \mathrm{m}$ ) with grain size $15 \pm 2$ and $28 \pm 3 \mathrm{~nm}$ were obtained by ball milling amorphous $\mathrm{NdFeB}$ powders and by annealing the powders at 500 and $700{ }^{\circ} \mathrm{C}$ for $30 \mathrm{~min}$, respectively. The recoil loops were then measured by fixing the powders in the epoxy. It was observed clearly that the experimental result agrees with the simulations that the single-phase magnets have open recoil loops and the openness decreases with increasing grain size. Similar results have also reported in $\mathrm{SmCo}_{5}$ magnets and $\mathrm{SmCo}_{5} / \alpha$-Fe nanocomposite magnets. ${ }^{24}$ Figure 2(b) compared the recoil loop openness of

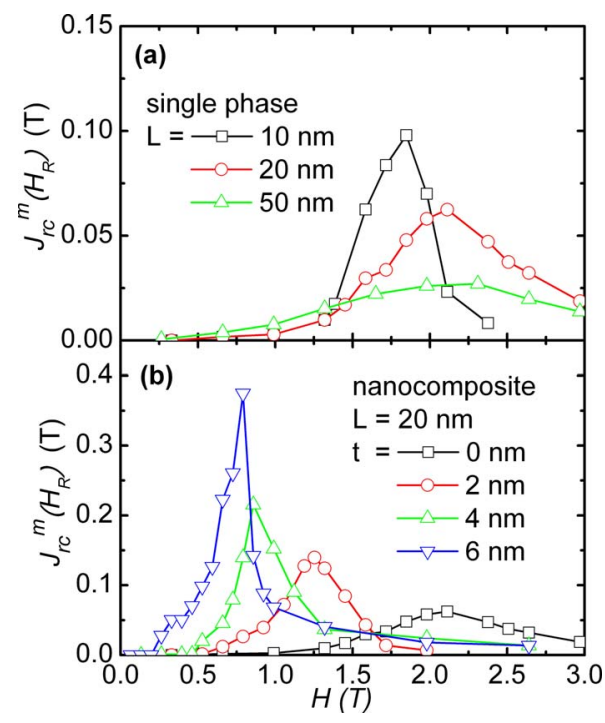

FIG. 2. (Color online) The dependence of $\Delta J_{\mathrm{rc}}^{m}\left(H_{R}\right)$ on the maximum reversal field $H_{R}$ for (a) the single phase magnets with different grain size and (b) the nanocomposite magnets with different soft-phase layer thickness while keeping $L=20 \mathrm{~nm}$.

the magnets with different thickness of the soft-phase layer. As one can see that the recoil loop openness increases with increasing soft-phase thickness (soft-phase content) for the nanocomposite magnets, which confirms that the soft phase contributes to the open recoil loops.

Given the contribution to recoil loop openness from grain boundaries and soft phases, further exploration of the nature of open recoil loops becomes desirable. What in common between the grain boundary effect and soft-phase effect is intergranular exchange interactions. Since the intergrain exchange coupling (either between the same phase, or between different phases) always leads to the deviation of magnetic moments in grain boundary regions from easy-axis directions of the grains, ${ }^{3,14-19}$ it is natural to assume that the exchange energy distribution over a nanocrystalline is related to the origin of open recoil loops. The static micromagnetic algorithm in this work is based on the minimization of Gibbs free energy, so we can calculate the energy distribution in the whole sample. Figures 4(a) and 4(b) show the exchange energy distribution along two adjacent hard-phase grains in a single-phase magnet and along the soft-phase layer in a nanocomposite magnet. It is very striking to see that the exchange energy increases very fast when approaching to a

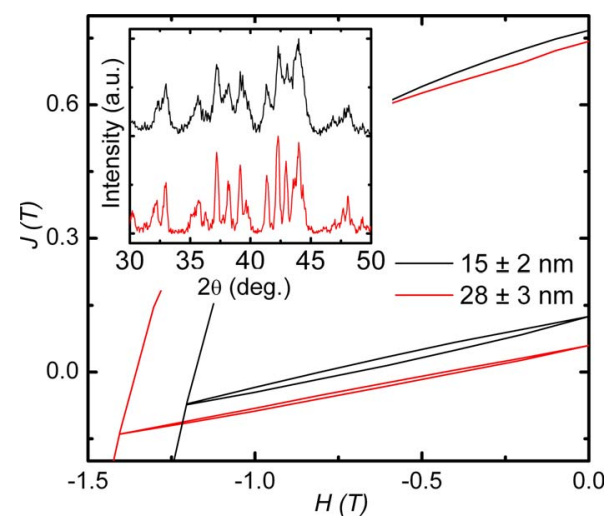

FIG. 3. (Color online) The experimental recoil loops of the $\mathrm{Nd}_{2} \mathrm{Fe}_{14} \mathrm{~B}$ single-phase magnets with different grain size. 

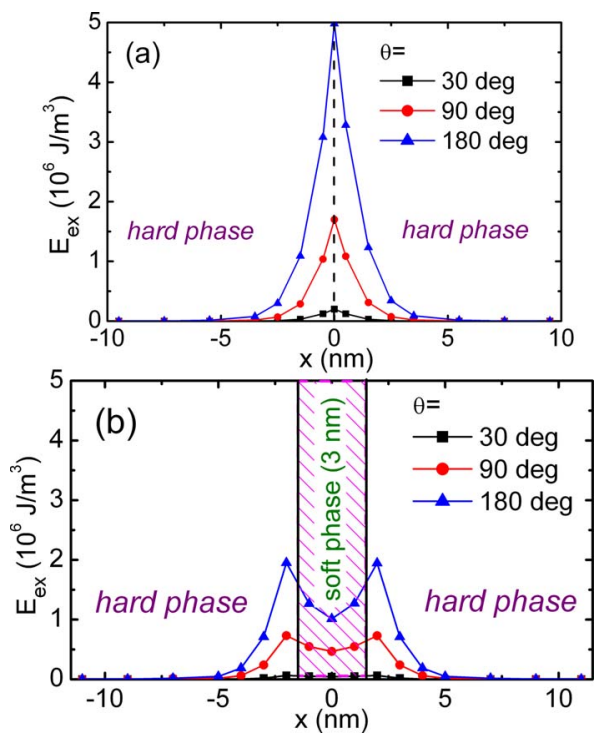

FIG. 4. (Color online) The exchange energy distribution along (a) two adjacent hard grains for the single-phase magnet and (b) two adjacent hard grains through the soft-phase layer for the nanocomposite magnet. $\theta$ is the angle between two easy-axis directions of the adjacent magnetically hard grains.

grain boundary for both single-phase and nanocomposite magnets, especially when two adjacent grains have large angle between two easy-axis directions. Surprisingly, the increase is much dramatic in case of single-phase magnets. It is not difficult to imagine that the high local exchange energy in grain boundary regions makes the magnetization configuration unstable.

One can better understand the origin of an open recoil loop by comparing the magnetization distributions in the single-phase and nanocomposite magnets [as shown in the insets of Figs. 1(a) and 1(b)]. First, the unstable magnetic moments in the grain boundary and/or soft-phase regions reverse under an applied relatively large external field of $H_{R}$. These moments keep reversed with removing external field unless the external field is small enough or is totally removed (lower branch of recoil loops). The unstable magnetic moments are now exchange-coupled with those in the adjacent hard grains if the external is removed. They will not reverse easily by reapplying a small external field (upper branch of recoil loops). Thus, an open recoil loop is formed owing to the irreversible magnetic moments in the grain boundary and/or in the soft-phase regions. The irreversible magnetization behavior is in turn due to the competition of exchange coupling between the adjacent hard grains which have large deviation angle between the stable magnetic moments in each grain core. Therefore, the more adjacent hard grains with large deviation angle are, the larger the recoil loop openness will be. This explains why the highest $\Delta J_{\mathrm{rc}}^{m}\left(H_{R}\right)$ value is always taking place when $H_{R}$ is at the coercive field. ${ }^{13,24}$ It is also important to mention that the magnetic moments in the soft phase may be more unstable by nature since the magnetocrystalline anisotropy of soft phase is much lower than that of hard phase even the exchange energy in the soft-phase region is lower than that in grain boundary region in single-phase hard magnets (as shown in Fig. 4). Hence, the contribution to the recoil loop openness from the grain boundary is less pronounced than that from the soft phase, given the grain size around $20 \mathrm{~nm}$.

In summary, it has been revealed through this investigation that the recoil loop openness is not only caused by inclusion of a soft magnetic phase in a nanocomposite magnet but also by grain boundaries. The latter was neglected in previous research on single-phase magnets because little attention was paid to nanocrystalline single-phase hard magnets. The magnetization distribution in a simulated magnet clearly shows that the openness of the recoil loops is attributed to magnetization reversal behavior in grain boundary and/or soft-phase regions, where the magnetic moments are unstable due to the high exchange energy.

This work was supported by U.S. DoD/MURI under Grant No. N00014-05-1-0497 and DARPA/ARO under Grant No. W911NF-08-1-0249.

${ }^{1}$ E. F. Kneller and R. Hawig, IEEE Trans. Magn. 27, 3588 (1991).

${ }^{2}$ S. D. Bader, Rev. Mod. Phys. 78, 1 (2006).

${ }^{3}$ C. B. Rong, H. W. Zhang, R. J. Chen, S. L. He, and B. G. Shen, J. Magn. Magn. Mater. 302, 126 (2006)

${ }^{4}$ E. H. Feutrill, P. G. McCormick, and R. Street, J. Phys. D 29, 2320 (1996).

${ }^{5}$ I. Panagiotopoulos, L. Withanawasam, and G. C. Hadjipanayis, J. Magn. Magn. Mater. 152, 353 (1996).

${ }^{6}$ D. Goll, M. Seeger, and H. Kronmuller, J. Magn. Magn. Mater. 185, 49 (1998).

${ }^{7}$ A. Bollero, O. Gutfleisch, K.-H. Muller, L. Schultz, and G. Drazic, J. Appl. Phys. 91, 8159 (2002).

${ }^{8}$ C. L. Harland, L. H. Lewis, Z. Chen, and B. M. Ma, J. Magn. Magn. Mater. 271, 53 (2004).

${ }^{9}$ K. Kang, L. H. Lewis, J. S. Jiang, and S. D. Bader, J. Appl. Phys. 98, 113906 (2005).

${ }^{10}$ C. B. Rong, V. Nandwana, N. Poudyal, Y. Li, J. P. Liu, Y. Ding, and Z. L. Wang, J. Phys. D 40, 712 (2007).

${ }^{11}$ Y. Choi, J. S. Jiang, J. E. Pearson, S. D. Bader, and J. P. Liu, Appl. Phys. Lett. 91, 022502 (2007).

${ }^{12}$ C. B. Rong, Y. Z. Liu, and J. P. Liu, Appl. Phys. Lett. 93, 042508 (2008).

${ }^{13}$ B. Zheng, H. W. Zhang, S. F. Zhao, J. L. Chen, and G. H. Wu, Appl. Phys. Lett. 93, 182503 (2008).

${ }^{14}$ R. Fischer, T. Schrefl, H. Kronumller, and J. Fidler, J. Magn. Magn. Mater. 153, 35 (1996)

${ }^{15}$ R. Fischer and H. Kronmuller, Phys. Rev. B 54, 7284 (1996).

${ }^{16}$ M. K. Griffiths, J. E. L. Bishop, J. W. Tucker, and H. A. Davies, J. Magn. Magn. Mater. 234, 331 (2001).

${ }^{17}$ C. B. Rong, H. W. Zhang, J. Zhang, X. B. Du, S. Y. Zhang, and B. G. Shen, J. Appl. Phys. 95, 1351 (2004).

${ }^{18}$ C. B. Rong, H. W. Zhang, X. B. Du, J. Zhang, S. Y. Zhang, and B. G. Shen, J. Appl. Phys. 96, 3921 (2004).

${ }^{19}$ C. B. Rong, H. W. Zhang, R. J. Chen, B. G. Shen, and S. L. He, J. Appl. Phys. 100, 123913 (2006).

${ }^{20}$ W. F. Brown, Jr., J. Phys. Soc. Jpn. Suppl. 17, B-I 540 (1962).

${ }^{21}$ X. Brunotte, G. Meunier, and J. F. Imhoff, IEEE Trans. Magn. 28, 1663 (1992).

${ }^{22}$ T. Schrefl, J. Fidler, and H. Kronmuller, J. Magn. Magn. Mater. 138, 15 (1994).

${ }^{23}$ R. Fischer, T. Schrefl, H. Kronmuller, and J. Fidler, J. Magn. Magn. Mater. 150, 329 (1995).

${ }^{24}$ C. B. Rong and J. P. Liu, J. Appl. Phys. 105, 07A714 (2009). 